\title{
CR-SUBMANIFOLDS OF TWO DIMENSIONAL COMPLEX PROJECTIVE SPACE
}

\author{
SHARIEF DESHMUKH AND M. A. AL-GWAIZ
}

1.

Among all submanifolds of a Kaehler manifold there are three typical classes:

the complex submanifolds, the totally real submanifolds and the CR-submanifolds. The notion of a CR-submanifold of a Kaehler manifold was introduced by Bejancu [1] and it includes the other two classes as special cases. If $\bar{M}$ is a Kaehler manifold with complex structure $J$ and $M$ is a submanifold of $\bar{M}, M$ is called a $C R$-submanifold of $\bar{M}$ if there exists a pair of orthogonal complementary distributions $D$ and $D^{\perp}$ on $M$ satisfying $J D=D$ and $J D^{\perp} \subset \nu$, where $\nu$ is the normal bundle of $M$.

It is known that every compact and orientable 3 -manifold $M$ possesses a contact structure, that is, $M$ carries a globally defined 1 -form $\eta$ with $\eta \wedge d \eta \neq 0$ everywhere on $M$ [4]. One can associate with $\eta$ a vector field $t$ determined by $\eta(t)=1$ and $d \eta(t, X)=0$ for all vector fields $X$ on $M$. If, in addition, $M$ is a Riemannian manifold with metric $g$ and $\eta$ satisfies $\eta(t)=g(X, t)$, then $\eta$ is called the contact metric stucture on $M$.

The object of the present paper is to study the 3-dimensional CR-submanifold of the 2-dimensional complex projective space $C P^{2}$. It is shown that a simply connected 3-dimensional CR-submanifold $M$ of $C P^{2}$ is either a contact manifold or a certain 2dimensional distribution on $M$ is integrable. We next consider those compact and simply connected CR-submanifolds which admit contact metric structure with respect to the induced metric, and prove that they are either diffeomorphic to $S^{3}$ or minimal submanifolds.

2.

Let $J$ be the almost complex structure and $g$ be the metric of constant holomorphic sectional curvature 4 on $C P^{2}$. If $\bar{\nabla}$ is the Riemannian connection on $C P^{2}$, then we have

$$
\left(\bar{\nabla}_{X} J\right)(Y)=0
$$

Let $M$ be a 3-dimentional CR-submanifold of $C P^{2}$. Then on $M$ there are two orthogonal complementary distributions, $D$ and $D^{\perp}$, satisfying $J D=D$ and $J D^{\perp}=T^{\perp} M$,

Received June 3, 1989.

1) This work has been supported by grant No. (Math/1409/05) of the Research Center, College of Science, King Saud University, Riyadh, Saudi Arabia. 
where $T^{\perp} M$ is the normal line boundle of $M$ (cf. [1]). It is clear that $\operatorname{dim} D=2$ and $\operatorname{dim} D^{\perp}=1$. Suppose $N$ is the unit normal vector field to $M$ and put $\xi=-J N$. Then $\xi$ is a globally defined unit vector field on $M$ which lies in $D^{\perp}$. We shall denote by $g$ both the metric on $C P^{2}$ and the induced metric on $M$. The Riemannian connection $\bar{\nabla}$ of $C: P^{2}$ induces a Riemannian connection $\nabla$ on $M$ and they are related by the formulae

$$
\bar{\nabla}_{X} Y=\nabla_{X} Y+h(X, Y), \bar{\nabla}_{X} J \xi=-A X, X, Y \in \mathcal{X}(M),
$$

where $h(X, Y)$ is the second fundamental form, $A$ is the Weingarten map and $\mathcal{X}(M)$ is the lie-algebra of vector fields on $M$. We also have the following relations for the hypersurface $M$ :

(2.3) $g(h(X, Y), J \xi)=g(A X, Y)$

(2.5) $R(X, Y ; Z, W)=\bar{R}(X, Y ; Z, W)+g(h(Y, Z), h(X, W)-g(h(X, Z), h(Y, W))$,

$$
X, Y, Z, W \in \mathcal{X}(M),
$$

where $R$ is the curvature tensor of $M$ and $\bar{R}$ is the curvature tensor of $C P^{2}$ given by

$$
\begin{gathered}
\bar{R}(X, Y) Z=g(Y, Z) X-g(X, Z) Y+g(J Y, Z) J X-g(J X, Z) J Y \\
+2 g(X, J Y) J Z, \quad X, Y, Z \in \mathcal{K}\left(C P^{2}\right) .
\end{gathered}
$$

If the trace of the second fundamental form $h(X, Y)$ is zero, then $M$ is called a minimal submanifold of $C P^{2}$.

3.

If we take $X \in D$, add the expansions of the equations $\left(\bar{\nabla}_{J X} J\right)(X)=0$ and $J\left(\bar{\nabla}_{X} J\right)(X)=$ 0 , and use (2.2) we get

$$
\nabla_{X} X+\nabla_{J X} J X+h(X, X)+h(J X, J X)+J[X, J X]=0, X \in D .
$$

Theorem 3.1 Let $M$ be a simply connected 3-dimensional CR-submanifold of $C P^{2}$. Then there exists a I-form $\alpha$ on $M$ which either defines a contact structure on $M$ or the distribution given by the kernel of $\alpha$ is integrable.

Proof. We note that for each unit vector field $e \in D,\{e, J e\}$ is an orthonormal local frame for $D$. Let $\left\{\omega^{1}, \omega^{2}\right\}$ be the dual frame of $\{e, J e\}$. We shall now show that the 2 -form $\omega^{1} \wedge \omega^{2}$ is independent of the frame $\{e, J e\}$. If $\{X, J X\}$ is another orthonormal frame for $D$ with dual frame $\{p, q\}$, then there exist smooth functions $f$ and $g$ such that, locally, we have $X=f e+g J e, J X=-g e+f J e$ and $f^{2}+g^{2}=1$. Consequently $p=f \omega^{1}+g \omega^{2}, q=-g \omega^{1}+f \omega^{2}$ and $p \wedge q=\omega^{1} \wedge \omega^{2}$. Taking the inner product with $\xi$ in (3.1), since $J \xi$ is normal, we get $g\left(\nabla_{X} X+\nabla_{J X} J X, \xi\right)=0, X \in D$. And 
since $\xi$ is a unit vector field we have $\xi \cdot g(\xi, \xi)=0$, that is, $g\left(\nabla_{\xi} \xi, \xi\right)=0$. For the orthonormal frame $\{e, J e, \xi\}$ on $M$, we get $d\left(\omega^{1} \wedge \omega^{2}\right)(e, J e, \xi)=0$, which means that the form $\omega^{1} \wedge \omega^{2}$ is closed. Since $M$ is simply connected the cohomology group $H^{2}(M, R)$ is trivial. Thus the closed 2-form $\omega^{1} \wedge \omega^{2}$ is exact and there exists a 1 -form $\alpha$ on $M$ such that $d \alpha=\omega^{1} \wedge \omega^{2}$. Now consider the set $U=\left\{p \in M:(\alpha \wedge d \alpha)_{p} \neq 0\right\}$, which is an open subset of $M$. $U$ is orientable as it has a non-vanishing 3 -form $\alpha \wedge d \alpha$. The 1-form $\alpha$ defines a contact structure on $U$, so there exists a vector field $t$ on $U$ determined by $\alpha(t)=1, d \alpha(t, X)=0$ for all vector fields $X$ on $U$ (cf. [2]). Since $d \alpha=\omega^{1} \wedge \omega^{2}$, it follows from $d \alpha(t, e)=0$ and $d \alpha(t, J e)=0$ that $\omega^{1}(t)=0$ and $\omega^{2}(t)=0$, that is, the vector field $t$ is parallel to $\xi$. On the other hand it is not difficult to see that $(\alpha \wedge d \alpha)_{p} \neq 0$ if and only if $\alpha_{p}(t)=1, \omega_{p}^{1}(t)=0$ and $\omega_{p}^{2}(t)=0$. For $\alpha_{p}(t)=1, \omega_{p}^{1}(t)=0$ and $\omega_{p}^{2}(t)=0$ imply that $\{t, e, J e\}$ are mutually orthogonal tangent vectors at $p$ and therefore $(\alpha \wedge d \alpha)_{p}(t, e, J e)=\left(\alpha \wedge \omega^{1} \wedge \omega^{2}\right)_{p}(t, e, J e)=1 \neq 0$. Hence $U=\left\{p \in M: \alpha_{p}(t)=1, \omega_{p}^{1}(t)=0, \omega_{p}^{2}(t)=0\right\}$ is a closed subset of $M$. Thus the set $U$ is both an open and a closed subset of $M$. $M$, being simply connected, is connected, and therefore either $U=M$ or $U$ is empty. When $U=M, \alpha$ defines a contact structure on $M$ and when $U$ is empty we have $\alpha \wedge d \alpha=0$ everywhere on $M$, which is the condition for integrability of the distribution $\{X \in \mathcal{X}(M): \alpha(X)=0\}$.

4.

In this section we study the simply connected 3-dimensional CR-submanifolds of $C P^{2}$ on which $\alpha$ defines a contact structure. Our main result is

Theorem 4.1 Let $M$ be a compact and simply connected 3-dimensional CRsubmanifold of $C P^{2}$. If the 1-form $\alpha$ defines a contact structure on $M$ which is also a contact metric structure with respect to the induced metric on $M$, then either $M$ is a minimal submanifold or $M$ is diffeomorphic to $S^{3}$.

Proof. Since $\alpha$ defines a contact metric structure on $M$ with respect to the induced metric structure on $M$, we have $\alpha(X)=g(X, t), X \in \mathcal{X}(M)$. From this it follows that $g(t, t)=1$, that is, $t$ is a unit vector field which is parallel to $\xi$, and thus $t=\xi$. If $\eta$ is a 1 -form dual to $\xi$, then we get $\alpha=\eta$. Since $d \eta=\omega^{1} \wedge \omega^{2}$, from $d \eta(\xi, e)=0$ and $d \eta(\xi, J e)=0$, we get $g\left(\nabla_{\xi} \xi, e\right)=0$ and $g\left(\nabla_{\xi} \xi, J e\right)=0$. As we already have $g\left(\nabla_{\xi} \xi, \xi\right)=0$ we conclude that $\nabla_{\xi} \xi=0$. Now using $(2.2)$ in $\left(\bar{\nabla}_{\xi} J\right)(\xi)=0$, we get $A \xi+J h(\xi, \xi)=0$. If we take $h(\xi, \xi)=\nu J \xi$, where $\nu$ is a smooth function, we obtain $A \xi=\nu \xi$, that is, $\xi$ is an eigenvector of $A$. The other two eigenvectors of $A$ will be from $D$. Suppose the two eigenvectors from $D$ are $e$ and $J e$ with $A e=\lambda e$ and $A J e=\mu J e$. Since the frame $\{e, J e, \xi\}$ diagonalizes $A$ we have $h(e, J e)=0, h(e, \xi)=0$ and $h(J e, \xi)=0$. Using (2.2) in $(\bar{\nabla} e J)(e)=0$ and taking the inner product with $J \xi$ we get $g\left(\nabla_{e} e, \xi\right)=0$. Similarly we get $g\left(\nabla_{J e} J e, \xi\right)=0$. Furthermore $\nabla_{\xi} \xi=0$ implies that $g\left(\nabla_{\xi} e, \xi\right)=0$ and $g\left(\nabla_{\xi} J e, \xi\right)=0$. Then, in view of the equations $\left(\bar{\nabla}_{e} J\right)(\xi)=0,\left(\bar{\nabla}_{J e} J\right)(\xi)=0$ and the structure equations 
of $M$, we have the following local equations

$$
\begin{aligned}
& \nabla_{e} e=a J e, \quad \nabla_{J e} J e=b e, \quad \nabla_{\xi} \xi=0 \\
& \nabla_{e} \xi=\lambda J e, \quad \nabla_{J e} \xi=-\mu e, \quad \nabla_{\xi} e=f J e \\
& \nabla_{\xi} J e=-f e, \quad \nabla_{e} J e=-a e-\lambda \xi, \quad \nabla_{J e} e=-b J e+\mu \xi,
\end{aligned}
$$

where $a, b, \lambda, \mu, \nu$ and $f$ are smooth functions.

Now consider the set $V=\{p \in M: \lambda \mu \nu \neq 0\}$. Since $V$ is an open subset of $M$ every point in $V$ has a neighbourhood where the equations (4.1) hold.

Equation (2.4) with different combinations of the frame vectors $\{e, J e, \xi\}$, in view of (4.1) and (2.6), gives

$$
f(\mu-\lambda)+\lambda(\nu-\mu)=-1, f(\mu-\lambda)+\mu(\lambda-\nu)=1, \nu(\lambda+\mu)=2(\lambda \mu-1)
$$

and

$$
\xi \cdot \lambda=0, \xi \cdot \mu=0, e . \nu=0, J e \cdot \nu=0, e \cdot \mu=b(\mu-\lambda), J e \cdot \lambda=a(\lambda-\mu) .
$$

Adding the first two equations in (4.2), we get $(\lambda-\mu)(\nu-2 f)=0$. Thus either $\lambda=\mu$ or $\nu=2 f$. Also from $d \eta=\omega^{1} \wedge \omega^{2}$, we get $-\eta([e, J e])=2$, and hence, using (4.1), we have $\lambda+\mu=2$. In case $\lambda=\mu$, we get $\lambda=\mu=1$ and, from (4.2), $\nu=0$, which does not occur on $V$. Thus on $V$ we have $\lambda \neq \mu$, and therefore $\nu=2 f$. To compute the values of $\lambda, \mu, \nu$ we note from (4.2) that $\nu=\lambda \mu-1$. Using the first two equations in (4.3), we get $\xi . \nu=0$, and, in view of (4.3), this implies that $\nu$ is constant.

Now solving the equations $\lambda+\mu=2$ and $\lambda-\mu=\sqrt{4-4 \lambda \mu}=2 \sqrt{-\nu}$, we get $\lambda=1+\sqrt{-\nu}$ and $\mu=1-\sqrt{-\nu}$. This shows that $\lambda$ and $\mu$ are both constants and, since $\lambda \neq \mu$, from (4.3) we get $a=0 b=0$. Using (2.5) and (2.6) together with (4.1) to compute $R(e, J e, J e, e)$, we get

$$
e . b+J e \cdot a=a^{2}+b^{2}+f(\lambda+\mu)+4+2 \lambda \mu .
$$

From the above equation we have $f+\nu+3=0$. Solving it with $\nu=2 f$, we get $\nu=-2$ and consequently $\lambda=1+\sqrt{2}, \mu=1-\sqrt{2}$. With the set $V=\{p \in M: \lambda \mu \nu=2\}$, and which is therefore closed, using the connectedness of $M$ we get either $V=M$ or $V$ is empty. When $V=M$, we have $\lambda+\mu+\nu=0$, that is $M$ is a minimal submanifold of $C P^{2}$, and when $V$ is empty we have $\lambda=1, \mu=1$ and $\nu=0$. If Ric denotes the Ricci tensor of $M$, then from (2.5) and (2.6) the Ricci tensor of $M$ is given by $\operatorname{Ric}(X, Y)=5 g(X, Y)+\operatorname{tr} \cdot A g(A X, Y)-g(A X, A Y)$. For $\lambda=1, \mu=1$ and $\nu=0$, we get $\operatorname{Ric}(e, e)=6, \operatorname{Ric}(J e, J e)=6, \operatorname{Ric}(\xi, \xi)=5 . \operatorname{Ric}(e, J e)=0, \operatorname{Ric}(J e, \xi)=0$. Thus for any non-zero vector field $X \in \mathcal{K}(M)$, we have locally $X=\omega^{1}(X) e+\omega^{2}(X) J e+\eta(X) \xi$, and

$$
\operatorname{Ric}(X, X)=\left[\omega^{1}(x)\right]^{2} \operatorname{Ric}(e, e)+\left[\omega^{2}(x)\right]^{2} \operatorname{Ric}(J e, J e)+[\eta(x)]^{2} \operatorname{Ric}(\xi, \xi)>0 .
$$

$M$ is therefore of strictly positive Rici curvature and, being compact and simply connected, by Hamiltons Theorem $M$ is diffeomorphie to $S^{3}$ (cf. [3]). 
Remark. We note that if $M$ is any real hypersurface of $C P^{2}$, then the unit normal vector field $N$ gives rise to the unit vector field $\xi=-J N$ on $M$. Then the kernel of the 1-form $\eta$ dual to $\xi$ gives rise to a smooth 2-dimensional distribution $D$ which satisfies $J D=D$, and $\xi$ spans the 1-dimensional distribution $D^{\perp}$ which satisfies $J D^{\perp}=T^{\perp} M$. Thus a real hypersurfaces of $C P^{2}$ is a 3-dimensional CR-submanifold of $C P^{2}$ and our theorems hold for the real hypersurface of $C P^{2}$. In a forthcoming paper we shall study 3-dimensional simply connected $\mathrm{CR}$-submanifolds of $C P^{2}$ on which $\alpha$ does not define the contact structure given by theorem 3.1.

\section{References}

[1] A. Bejancu, "CR-submanifolds of a Kachler manifold-I," Proc. A mer Math. Soc. 69 (1978), 134-142.

[2] D. E. Blair, Contact manifolds in Riemannian Geometry, Lecture Notes in Math. 509, Springer Verlag, Berlin and New York 1976.

[3] R. S. Hamilton, "Three manifolds with positive Ricci curvature," J. Diff. Geom. 17 (1982), 255-306.

[4] J. Martinet, Formés de contact sur lés variétés de diménsion 3, Proc. Liverpool Singularities Symp. II, Springer lecture notes in Math. 209 (1971), 142-163.

Department of Mathematics, College of Science, King Saud University, POB 2455, Riyadh-11451, Saudi Arabia. 